\title{
Development of a GC/MS method for the qualitative and quantitative analysis of mixtures of free fatty acids and metal soaps in paint samples
}

\author{
Dr. Jacopo La Nasa \\ Department of chemistry and industrial chemistry, University of Pisa \\ Via Giuseppe Moruzzi 13 \\ 56124, Pisa, Italy \\ jacopo.lanasa@for.unipi.it
}

Prof. Francesca Modugno

Department of chemistry and industrial chemistry, University of Pisa

Via Giuseppe Moruzzi 13

56124, Pisa, Italy

francesca.modugno@for.unipi.it

\section{Matteo Aloisi}

Department of chemistry and industrial chemistry, University of Pisa

Via Giuseppe Moruzzi 13

56124, Pisa, Italy

matteoaloisi2610@gmail.com

\section{Dr. Anna Lluveras Tenorio}

Department of chemistry and industrial chemistry, University of Pisa

Via Giuseppe Moruzzi 13

56124, Pisa, Italy

anna.lluveras@for.unipi.it

\section{Prof. Ilaria Bonaduce*}

Department of chemistry and industrial chemistry, University of Pisa Via Giuseppe Moruzzi 13

56124, Pisa, Italy 
Ilaria.bonaduce@unipi.it

phone: +39 0502219328

*corresponding author 


\section{Keywords}

Gas chromatography/mass spectrometry

Metal soaps

Free fatty acid

Silylation

Hexamethyldisilazane

$\mathrm{N}, \mathrm{O}$-bis(trimethylsilyl)trifluoroacetamide

Painting

\section{Abstract}

In this paper we present a new analytical GC/MS method for the analysis of mixtures of free fatty acids and metal soaps in paint samples. This approach is based on the use of two different silylating agents: N,Obis(trimethylsilyl)trifluoroacetamide (BSTFA) and 1,1,1,3,3,3-hexamethyldisilazane (HMDS).

Our experimentation demonstrated that HMDS does not silylate fatty acid carboxylates, so it can be used for the selective derivatization and GC/MS quantitative analysis of free fatty acids. On the other hand BSTFA is able to silylate both free fatty acids and fatty acids carboxylates. The reaction conditions for the derivatization of carboxylates with BSTFA were thus optimized with a full factorial $3^{2}$ experimental design using lead stearate and lead palmitate as model systems. The analytical method was validated following the ICH guidelines. The method allows the qualitative and quantitative analysis of fatty acid carboxylates of sodium, calcium, magnesium, aluminium, manganese, cobalt, copper, zinc, cadmium, and lead and of lead azelate. In order to exploit the performances of the new analytical method, samples collected from two reference paint layers, from a gilded 16th century marble sculpture, and from a paint tube belonging to the atelier of Edvard Munch, used in the last period of his life (1916-1944), were characterized. 


\section{Introduction}

Metal soaps are mono or poly-carboxylates of fatty acids and alkali, alkaline earth or transition metals. Thanks to their emulsifying properties, lubricity and hydrophobicity, metal carboxylates are valuable in a variety of industrial applications, and are present in the formulations of a wide range of commercial products.

Depending on the nature of the cation, on the alkyl chain length, and on the presence or absence of unsaturations, the physical properties and applications of metal carboxylates may vary considerably [1]. The most commonly used metal soaps are those produced from stearic (octadecanoic) acid. Sodium, potassium, calcium, magnesium, zinc and aluminium are the most frequently used metals. Metal soaps are used as stabilizers, emulsifiers, dryers, plasticizers, lubricants, thickening and anti-precipitation agents in many manufacturing processes, including the cosmetic and pharmaceutical field, the dry cleaning processes, rubber industry, and in paint formulations [2-4].

In oil paint formulations metal soap have commonly been added as surfactant additives to facilitate the dispersion of pigments into the medium. Dispersing agents were added to paints since the $19^{\text {th }}$ century, and aluminium and zinc stearates were patented as dispersion agents and widely used after $1920[3,5,6]$. Metal soaps are also known to be spontaneously formed in artistic oil paintings, as a product of reactions between the excess of carboxylic groups, released by the hydrolysis of triglycerides as well as those produced by the oxidative cleavage of unsaturated fatty acids contained in oil media, and the cations contained in certain pigments, such as lead and zinc [7-12]. The processes leading to the formation of metal soaps in paint films is a concern for conservators, as they can heavily affect the visual appearance of an artwork [11]. Metal soaps have the tendency to aggregate, and they may lead to the formation of inclusions, which can sometimes form lumps or protrusions on the paint surface, or can migrate from a paint layer to an adjacent one. Moreover metal soaps can catalyze the hydrolysis of the acylglycerols of the paint medium, leading to a deterioration of the painted surfaces $[13,14]$.

The possibility of qualitatively and quantitatively determine fatty acid carboxylates of metal soaps in complex matrices, and to distinguish them from free fatty acids, is thus valuable in the field of conservation science, to investigate alteration processes, to assess formulations, and to establish relations between formulations, environmental parameters and alteration of the paint surfaces.

X-ray powder diffraction pattern analysis (XRD) allows the identification of metal soaps by achieving information about their crystal structure $[1,15]$. Fourier transform infrared spectroscopy (FTIR), Raman spectroscopy and NMR spectroscopy can be used to indicate the presence of metal soaps by the detection of their characteristic absorption bands and to understand their formation and crystallization processes [16-23]. When complex mixtures of acylglycerols, free fatty acids, and carboxylates are present, though, spectral features can be difficult to interpret and to achieve information at the molecular level. Recently a 
derivatization reaction with sulphur tetra fluoride $\left(\mathrm{SF}_{4}\right)$ has been proposed to differentiate carboxylates and free fatty acids from esters and keto groups by FTIR spectroscopy [24].

Gas chromatography/mass spectrometry (GC/MS) is potentially a suitable technique for the molecular identification and quantification of fatty acid carboxylate anions in metal soaps. One of the main drawbacks of this approach is the extraction step(s), as metal soaps are highly insoluble in most solvents: their analysis can be achieved after acidification, to transform carboxylates into acids [25-28]. This approach, though, does not permit then to separately determine free fatty acids and metal soaps.

In this paper we present the development and optimization of a simple analytical approach for the qualitative and quantitative characterisation of mixtures of free fatty acids and of metal soaps in paint samples by GC/MS analysis. To this aim we exploited the different reactivity of two silylating agents, N,Obis(trimethylsilyl)trifluoroacetamide (BSTFA), and 1,1,1,3,3,3-hexamethyldisilazane (HMDS), to achieve selective solubilisation and derivatization of fatty acid carboxylates and free fatty acids. The proposed analytical approach permits to qualitatively determine and quantify fatty acid carboxylates and free fatty acids in paint samples. The method was set up and subsequently used to successfully characterise two model paint layers containing a drying oil and a pigment [29], a gilding sample from a $16^{\text {th }}$ century marble sculpture by an anonymous artist from Lazio, Italy, and a sample from paint tube belonging produced by Winsor \& Newton to the atelier of Edvard Munch (Ekely, Oslo), used in the last period of his life (19161944) 


\section{Materials and methods}

\subsection{Chemicals}

Bidistilled water, absolute ethanol, acetone, diethyl ether, $n$-hexane and iso-octane (HPLC grade), lead acetate, manganese chloride, copper acetate, cobalt acetate, lead nitrate, and stearic acid (purity >99\%), hexadecane, tridecanoic, hexadecanoic and octadecanoic acid, (purity 99\%), (N,Obis(trimethylsilyl)trifluoroacetamide (BSTFA) containing 1\% trimethylchlorosilane (TMCS) and 1,1,1,3,3,3hexamethyldisilazane (HMDS) were all purchased from Sigma-Aldrich (U.S.A.).

Aluminium stearate was purchased from Kremer Pigmente, GmbH \& Co. (Aichstetten, Germany). Zinc stearate containing the $25 \%$ of zinc palmitate, manganese stearate containing the $25 \%$ of manganese palmitate, calcium stearate (tech. grade), and sodium stearate (tech. grade) were purchased from SigmaAldrich. Cadmium palmitate was provided by Prof. Leslie Carlyle. Copper, cobalt, and manganese stearates and lead azelate were synthesized according to the procedures summarised in section 2.2. Standard solutions were prepared in acetone (tridecanoic acid: azelaic acid $4.06 \mu \mathrm{g} / \mathrm{g}$, palmitic acid: $4.39 \mu \mathrm{g} / \mathrm{g}$, and stearic acid: $6.62 \mu \mathrm{g} / \mathrm{g})$.

\subsection{Synthesis of metal soaps}

The synthesis of metal stearates was adapted from $[17,30]$. Lead, cobalt, copper, cadmium and manganese stearates were synthesized by adding an aqueous solution ( $0.4 \mathrm{M}$ ) of metal salt (lead acetate, manganese chloride, copper acetate, cobalt acetate, lead nitrate) to an ethanolic solution of stearic acid $(0.2 \mathrm{M})$ at $70^{\circ} \mathrm{C}$ with continuous stirring for 30 minutes. The precipitates were washed with warm water, acetone and diethyl ether in order to remove the salt excess and the un-reacted free fatty acids.

For the synthesis of lead azelate alcoholic solutions of lead nitrate and azelaic acid were mixed at $40^{\circ} \mathrm{C}$. After mixing the resulting solution was covered with a watch glass and left in the water bath to return to ambient temperature. After 12 hours the crystallized lead azelate was removed from the solution and washed with warm water, acetone and diethyl ether in order to remove the salt excess and un reacted free fatty acids.

Assuming that all the residues of fatty acids were removed in the washing step (hot water, acetone and diethyl ether), the weight content of fatty acid carboxylates in all the synthesized metal carboxylate were determined following the procedure reported in [31]. Briefly, 10-40 $\mu \mathrm{g}$ of sample were subject to acidification with of hydrochloridric acid for $10 \mathrm{~min}$ in ultrasonic bath at $60^{\circ} \mathrm{C}$. The carboxylic acids were then extracted using $400 \mu \mathrm{L}$ diethyl ether for three times. The extracted solutions were admixed, an aliquot was evaporated to dryness under nitrogen stream, and subject to derivatization for the GC/MS analysis following the conditions reported in Section 2.4.2. As an example, the chromatogram obtained from the analysis of cobalt stearate is reported as Figure S.1 in the supplementary materials. 


\subsection{Samples}

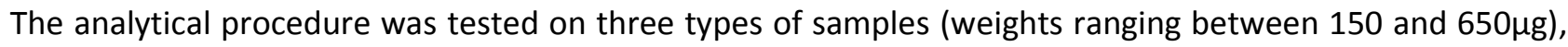
with matrixes of increasing complexity.

- two reference paint layers provided by Prof. Leslie Carlyle, made in 1999 as part of the MOLART Program [29]. These contained linseed oil as binder and two different pigments, lead white and vine black. Samples were naturally aged and preserved in laboratory at room temperature and RH\%.

- a gilding sample from a $16^{\text {th }}$ century marble sculpture by an anonymous artist from Lazio; the full chemical composition of the sample was determined by analytical pyrolysis (Py) coupled with GC/MS and infrared spectroscopy and the results are reported in the supplementary material in section S.2. The object was in good conservation conditions, and the sample, before analysis, was stored in a glass vial for chromatography.

- a paint tube belonging to the atelier of Edvard Munch (Ekely, Oslo), used in the last period of his life (1916-1944), was analysed. The tube was produced by Winsor \& Newton and labelled "ultramarin dunke". The chemical composition of the sample was determined by liquid chromatography coupled with mass spectrometry, Py-GC/MS, and infrared spectroscopy, and results have been published elsewhere [5, 32]. The object was in good conservation conditions, the paint still liquid, and the sample, before analysis, was stored in a glass vial for chromatography.

\subsection{Sample treatment}

\subsubsection{Derivatization of metal soaps and free fatty acids with BSTFA containing $1 \%$ trimethylchlorosilane}

Metal soaps, in the range of $10-40 \mu \mathrm{g}$, were added with $50 \mu \mathrm{L}$ of the tridecanoic acid solution (internal standard for derivatization). The mixture was dried under nitrogen flow at room temperature, and subsequently added with $200 \mu \mathrm{L}$ of BSTFA and $1500 \mu \mathrm{L}$ of iso-octane. $50 \mu \mathrm{L}$ of the hexadecane solution were added just before injection (internal standard for injection). Samples were derivatized according to the conditions described in the text for the optimisation of the method. When standard solutions or samples from the paints were analysed, they were added with $5 \mu \mathrm{L}$ of the tridecanoic acid solution. The mixture was dried under nitrogen flow at room temperature, and subsequently added with $20 \mu \mathrm{L}$ of BSTFA and $150 \mu \mathrm{L}$ of iso-octane. $5 \mu \mathrm{L}$ of the hexadecane solution were added just before injection. The reaction time and temperature were set at $81 \mathrm{~min}$ and $78^{\circ} \mathrm{C}$, as result of the optimisation described in the experimental section. 


\subsubsection{Derivatization of free fatty acids with HMDS}

Samples (100-200 $\mu \mathrm{g})$ were added with $5 \mu \mathrm{L}$ of tridecanoic acid solution, and then dried under nitrogen flow at room temperature in order to remove the solvent. The residual solid was subjected to derivatization for the GC/MS analysis with $20 \mu \mathrm{L}$ of HMDS, $150 \mu \mathrm{L}$ of iso-octane at $60^{\circ} \mathrm{C}$ for $30 \mathrm{~min} .5 \mu \mathrm{L}$ of hexadecane solution were added just before injection as injection internal standard [31, 33].

\subsection{Gas Chromatography Mass spectrometry}

GC/MS instrumentation consisted of an Agilent Technologies 6890N Gas Chromatograph coupled with a 5973 Mass Selective Detector single-quadrupole mass spectrometer.

Samples were injected in splitless mode at $280^{\circ} \mathrm{C}$. GC separation was performed on a fused silica capillary column HP-5MS (J\&W Scientific, Agilent Technologies, stationary phase 5\% diphenyl-95\% dimethylpolysiloxane, $30 \mathrm{~m}$ length, $0.25 \mathrm{~mm}$ i.d., $0.25 \mu \mathrm{m}$ film thickness). Chromatographic conditions were [5, 6]: initial temperature $80^{\circ} \mathrm{C}, 2 \mathrm{~min}$ isothermal, $20^{\circ} \mathrm{C} / \mathrm{min}$ up to $280^{\circ} \mathrm{C}, 10 \mathrm{~min}$ isothermal.

MS parameters: electron impact ionization ( $\mathrm{El}, 70 \mathrm{eV}$ ) in positive mode; ion source temperature $230^{\circ} \mathrm{C}$; chromatograms were acquired simultaneously in full scan (range 50-700 m/z) and selected ion monitoring (SIM) modes; interface temperature $280^{\circ} \mathrm{C}$. The injection volume was $2 \mu \mathrm{L}$.

The GC/MS quantitative analysis of palmitic acid and stearic acid was performed using calibration curves calculated on the basis of SIM chromatograms. The selected ions for the SIM acquisition were: azelaic acid $\mathrm{m} / \mathrm{z} 129$ and 317, palmitic acid m/z 117 and 313, stearic acid m/z 117 and 341 . 


\section{Results and discussion}

Free fatty acids are quite easily silylated by most silylating agents [34]. We tested the reactivity of both HMDS and BSTFA with metal soaps and free fatty acids, in order to evaluate the possibility to selectively quantify the two species in mixtures, exploiting the different reactivity of the two reagents. HMDS is a weak TMS donor, while BSTFA, especially when added with catalytic amounts of TMCS, is one of the most potent silylating agents for the analytical derivatization of a large number of $\mathrm{X}-\mathrm{H}$ acidic compounds [34]. A previous work showed that BSTFA is also able to silylated lead stearates, although reaction yields were not discussed [26].

The silylation reaction is viewed as a nucleophilic attack by the more electronegative heteroatom, oxygen in our case, upon the silicon atom of the silyl donor [35]. In principle silylation of carboxylates should thus be possible, although the reaction yield will be dependent on several factors. First of all the solubility of the metal soap may be expected to play a fundamental role: if the metal soap is not soluble in the reaction medium, the reaction may be slowed down. The nature of the bond can also be expected to be another important factor. A lower reactivity may be expected when the bond between the carboxylate and the metal is covalent with respect to when it is ionic. As it has been shown that lead carboxylates are characterised by bonds with a substantial covalent character[36], we thus decided to start our studies by investigating the reactivity of lead soaps.

\subsection{Reactivity of free fatty acids and lead soaps toward HMDS and BSTFA}

A set of standard solutions containing $4.0 \mathrm{ppm}$ of the free fatty acid hexadecanoic acid (palmitic acid) and octadecanoic acid (stearic acid) were derivatized with HMDS and BSTFA, according to the conditions reported in section 2.4.2 and reported in the literature[31]. The areas of the chromatographic peaks obtained for the same compound with the two derivatizing agents were compared using a t test (C.I. $99 \%$ ), and resulted to belong to the same statistical populations: free fatty acids reacted both with HMDS and BSTFA, with the same reaction yields.

Lead carboxylates $(5-20 \mu \mathrm{g})$ were silylated by BSTFA, but they did not react with HMDS, as the chromatograms of the HMDS derivatives showed signals relative to the silylesters of fatty acids at the blank level (Figure S.1). Moreover, when HMDS was added to lead soaps, the solid was still visible, indicating that lead soaps do not dissolve in the reaction medium, while when BSTFA was added, a clear solution was obtained.

\subsection{Optimisation of the reaction conditions for the silylation of metal carboxylates with BSTFA}

With the aim of maximizing the yield of silylation of fatty acid lead carboxylates with BSTFA, the reaction conditions of lead stearate and lead palmitate were optimized varying temperature and reaction time. 
Experiments were set up according to a three levels full factorial design [37]. The values of the levels explored for the two factors were 60,90 and $120 \mathrm{~min}$ for the reaction time and $60^{\circ} \mathrm{C}, 80^{\circ} \mathrm{C}$ and $100^{\circ} \mathrm{C}$ for the temperature. The chosen starting values of temperature and time were based on the procedure used for the silylation of free fatty acids reported in the literature [31]. The maximum value of temperature was chosen taking into consideration the boiling temperature of the solvent $\left(99^{\circ} \mathrm{C}\right)$, and of BSTFA $\left(155^{\circ} \mathrm{C}\right)$. The reaction time at $120 \mathrm{~min}$ showed a decrease of the signals, so longer reaction times were not investigated. The chromatographic areas of trimethylsilyl esters of palmitic and stearic acid were used as experimental response $(R)$, after normalisation for the sample concentration. For each of the two trimethylsilylesters the response was modelled as a function of time $(t)$ and temperature $(T)$ according to the following model function $R=b_{0}+b_{1} t+B_{2} T+b_{3} t T+b_{4} t^{2}+b_{5} T^{2}$ [37]. All experiments were carried out in triplicates, and the response values, corresponding to the average of the three replicates, are reported in Table 1. 
Table 1- Experimental matrix containing the levels used for the full factorial experimental design used in the optimisation of the derivatization reaction of lead palmitate and lead stearate with BSTFA and relative experimental responses. The chromatographic areas of trimethylsilyl esters of palmitic and stearic acid were used as experimental response (R), after normalisation for the sample concentration. The experiments were performed in random order, and each experiment was carried out in three replicates. The average response of the replicates are reported together with their coefficient of variation (CV \%)

\begin{tabular}{|c|c|c|c|c|c|c|c|c|}
\hline Experiment & Time $t$ (min.) & Temperature $T\left({ }^{\circ} \mathrm{C}\right)$ & $t($ coded $)$ & $T$ (coded) & $\begin{array}{c}\text { R palmitic acid } \\
\text { (average) }\end{array}$ & $\begin{array}{c}\text { R palmitic acid } \\
\text { CV\% }\end{array}$ & $\begin{array}{c}\text { R stearic acid } \\
\text { (average) }\end{array}$ & $\begin{array}{c}\text { R stearic acid } \\
\text { CV\% }\end{array}$ \\
\hline 1 & 60 & 60 & -1 & -1 & 243 & 4 & 136 & 20 \\
\hline 2 & 60 & 80 & -1 & 0 & 473 & 16 & 276 & 14 \\
\hline 3 & 60 & 100 & -1 & 1 & 330 & 4 & 186 & 14 \\
\hline 4 & 90 & 60 & 0 & -1 & 293 & 17 & 216 & 24 \\
\hline 5 & 90 & 80 & 0 & 0 & 462 & 15 & 269 & 11 \\
\hline 6 & 90 & 100 & 0 & 1 & 337 & 16 & 208 & 6 \\
\hline 7 & 120 & 60 & 1 & -1 & 93 & 18 & 78 & 19 \\
\hline 8 & 120 & 80 & 1 & 0 & 260 & 20 & 135 & 23 \\
\hline 9 & 120 & 100 & 1 & 1 & 94 & 17 & 14 & 16 \\
\hline
\end{tabular}


The calculation of the coefficients was done with the open source software R Studio Version 1.0.136 and R version 3.3.2 (The R Foundation for Statistical Computing) with Chemometrics with $R$ package, using the coded values of the variables $(-1 ; 0 ; 1$, as shown in Table \$1). The coefficients obtained for the model are reported in Table 2 and the two response surfaces are shown in Figure 1.

Table 2. - Coefficients for the model of the response $\left(R=b_{0}+b_{1} t+B_{2} T+b_{3} t T+b_{4} t^{2}+b_{5} T^{2}\right)$ of palmitate and stearate in dependence of time $(t)$ and temperature $(T)$, calculated from the experimental matrix reported in Table S1. Predicted vs. experimental responses for the derivatization at $80^{\circ} \mathrm{C}$ for 80 minutes are also reported, along with $\mathrm{CV} \%$ (intraday, $\mathrm{N}=4$ )

\begin{tabular}{c|c|c}
\hline Coefficients (b) & Palmitate & Stearate \\
\hline $\mathbf{b}_{\mathbf{0}}$ & 474.41 & 287.03 \\
\hline $\mathbf{b}_{\mathbf{1}}$ & 22.25 & -4.00 \\
\hline $\mathbf{b}_{\mathbf{2}}$ & -100.13 & -61.99 \\
\hline $\mathbf{B}_{\mathbf{3}}$ & -21.25 & -28.83 \\
\hline $\mathbf{B}_{\mathbf{4}}$ & -168.72 & -88.3 \\
\hline $\mathbf{B}_{5}$ & -113.62 & -90.7 \\
\hline \hline Predicted response & 477 & 289 \\
\hline Experimental response & $454 \pm 28$ & $288 \pm 40$ \\
\hline $\mathbf{C V} \%$ & 7.2 & 7.6 \\
\hline \multicolumn{2}{|c}{}
\end{tabular}

a)

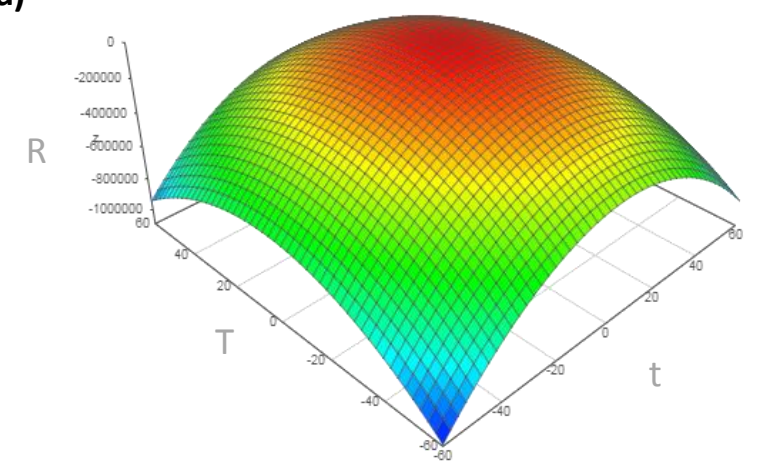

b)



Figure 1 - Response surfaces for the reaction of lead palmitate (a) and lead stearate (b) with BSTFA in function of coded temperature $(T)$ and time $(t)$. The maximum of the coded values were $(T, t)=(0.03,-0.35)$ for the lead palmitate and $(T, t)=(0.09,-0.45)$ for lead stearate, corresponding to $83^{\circ} \mathrm{C} / 80 \mathrm{~min}$ and $79^{\circ} \mathrm{C} / 74 \mathrm{~min}$ respectively. The maximum were calculated using the software Wolfram Alpha [38]

The maxima of the two functions were calculated, and the average values of the two maxima resulted $81^{\circ} \mathrm{C}$ for 78 minutes [38]. To validate the model, the reaction was performed again in triplicates at the optimal conditions using calibration curves: the predicted and the experimental results did not show statistically significant differences at $95 \%$ confidence level. As reported in Table 3, at the optimal conditions, the derivatization of lead stearate and lead palmitate is quantitative. 
Table 3 - Derivatization yields ${ }^{1}$ of metal soaps with BSTFA and relative coefficient of variation (CV \%), according to the experimental conditions set-up in this work

\begin{tabular}{c|c|c}
\hline Metal carboxylate & Derivatization yield & CV \% \\
\hline Sodium stearate & $95 \%$ & 7 \\
\hline Calcium stearate & $99 \%$ & 4 \\
\hline Magnesium palmitate & $104 \%$ & 8 \\
\hline Magnesium stearate & $93 \%$ & 8 \\
\hline Aluminium stearate & $99 \%$ & 4 \\
\hline Manganese stearate & $105 \%$ & 2 \\
\hline Cobalt stearate & $105 \%$ & 1 \\
\hline Copper stearate & $107 \%$ & 2 \\
\hline Zinc palmitate & $104 \%$ & 8 \\
\hline Zinc stearate & $96 \%$ & 10 \\
\hline Cadmium palmitate & $108 \%$ & 2 \\
\hline Lead palmitate & $98 \%$ & 9 \\
\hline Lead stearate & $109 \%$ & 3 \\
\hline Lead azelate & $91 \%$ & 12 \\
\hline
\end{tabular}

Azelaic acid is the main oxidation product deriving from the oxidation of a drying oil, and it can form metal soaps [39]. The derivatization of lead azelate with BSTFA using the optimised reaction conditions was thus tested, showing a reaction yield above $90 \%$ (Table 3). Finally, as the reaction conditions were optimised on lead soaps, the procedure was tested on aluminium, cadmium, cobalt, copper, manganese, magnesium, and zinc carboxylates in order to evaluate the effect of the different cations on the reaction yield. Reaction yields were all above 90\%, with CV\% below $10 \%$ (Table 3).

Finally, tripalmitin together with a standard mixture of metal carboxylate was subject to the derivatization procedure using both HMDS and BSTFA, in order to evaluate if transesterification of glycerides could occur in the experimental conditions adopted. Transesterification would in fact affect the analyses when metal soaps and free fatty acids are present together with glycerides in a sample. The chromatographic peaks ascribable to trimethylsilyl palmitate showed an area below the area corresponding to the detection limit. Based on these results, we conclude that it is possible to qualitatively and quantitatively determine mixtures of free fatty acids and metal soaps by dividing the sample into two aliquots, one to be derivatized with HMDS, in order to determine free fatty acids, and the other to be derivatized with BSTFA, in order to determine the sum of free fatty acids and fatty acid carboxylates, as shown in Figure 2.

\footnotetext{
${ }^{1}$ Derivatization yields were calculated on the basis of purities of metal carboxylates reported by the manufacturers, or the content of fatty acid carboxylates in the synthesised metal soaps evaluated as described in paragraph 2.2 .
} 


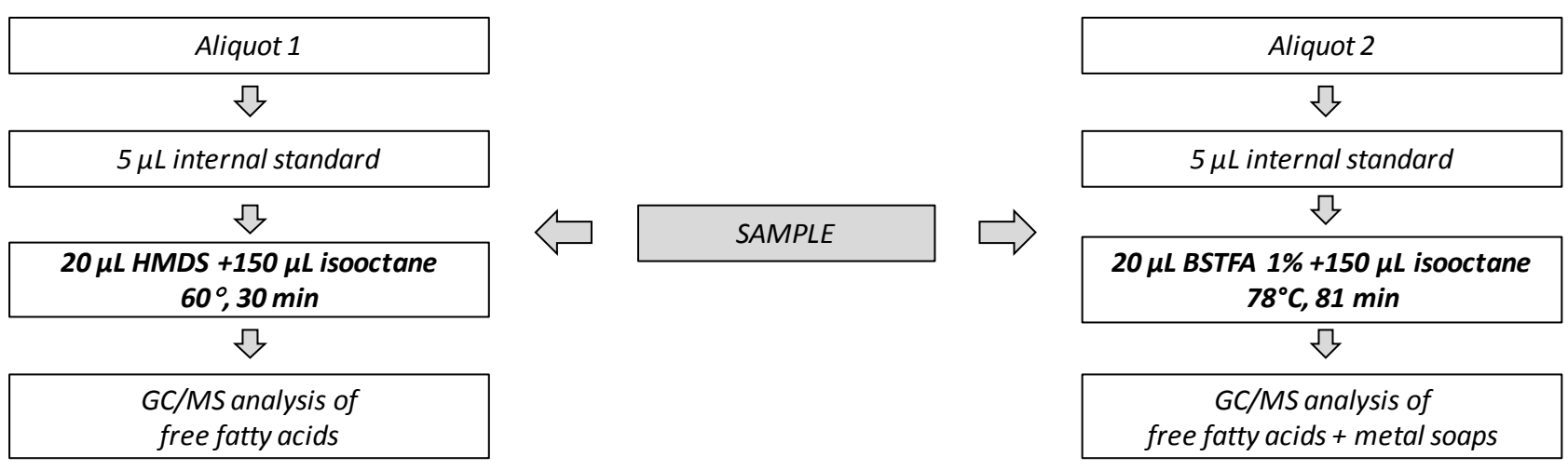

Figure $\mathbf{2}$ - Analytical procedures for the characterization of free fatty acids and metal soap by GC/MS analysis

\subsection{Validation of the procedure}

After defining the best reaction conditions, we performed the full validation of the silylating procedure of BSTFA for lead carboxylates, and of HMDS for free fatty acids according to the ICH guidelines [40]. Limits of detection (LOD), limits of quantitation (LOQ), reproducibility, recovery, intraday and interday precision were determined. Validation parameters are reported in Table 4.

In order to exploit the method for quantitative analysis, calibration curves were also determined in the range $2-25 \mu \mathrm{g} / \mathrm{g}$ using six standard solutions at different concentration. The areas were normalized using the response of the internal standard and plotted versus the concentration performing a least squares adjustment. The $R^{2}$ coefficients obtained for all the standards were in the range 0.9961-0.9992. The limits of detection (LOD) and quantitation (LOQ) are reported in Table 4, and were determined based on the standard deviation of replicate blank samples and on the slope of the calibration curves. Limits of detection were below $0.4 \mu \mathrm{g} / \mathrm{g}$ and limits of quantitation were below $0.5 \mu \mathrm{g} / \mathrm{g}$. The intra-day and inter-day RSD\% on the analytical response were lower than 3.5 and 5.9 for the BSTFA procedure, and lower than 2.7 and 6.1 for the HMDS approach. 
Table 4 - Validation parameters of the derivatization reactions

\begin{tabular}{|c|c|c|c|c|c|c|c|c|c|c|}
\hline & \multirow{2}{*}{ Compound } & \multicolumn{2}{|c|}{ Reaction conditions } & \multicolumn{2}{|c|}{ Calibration curves } & \multirow{2}{*}{$r^{2}$} & \multirow{2}{*}{$L O D(\mu g / g)$} & \multirow{2}{*}{$\operatorname{LOQ}(\mu \mathrm{g} / \mathrm{g})$} & \multirow{2}{*}{ CV \% intra } & \multirow{2}{*}{ CV \% inter } \\
\hline & & Time (min.) & Temperature $\left({ }^{\circ} \mathrm{C}\right)$ & Slope & Intercept & & & & & \\
\hline \multirow{3}{*}{  } & Azelaic acid & \multirow{3}{*}{81} & \multirow{3}{*}{78} & 0.5 & -0.01 & 0.9965 & 0.1 & 0.2 & 3.2 & 5.6 \\
\hline & Palmitic acid & & & 1.1 & 1.3 & 0.9992 & 0.2 & 0.3 & \multirow{2}{*}{3.5} & \multirow{2}{*}{5.9} \\
\hline & Stearic acid & & & 0.8 & 1.4 & 0.9974 & 0.3 & 0.4 & & \\
\hline \multirow{3}{*}{$\stackrel{n}{\text { 呈 }}$} & Azelaic acid & \multirow{3}{*}{30} & \multirow{3}{*}{60} & 0.4 & -0.1 & 0.9961 & 0.1 & 0.2 & 2.5 & 5.7 \\
\hline & Palmitic acid & & & 1.2 & -2.1 & 0.9998 & 0.2 & 0.3 & \multirow{2}{*}{2.7} & \multirow{2}{*}{6.1} \\
\hline & Stearic acid & & & 1.1 & -2.7 & 0.9992 & 0.4 & 0.5 & & \\
\hline
\end{tabular}




\subsection{Paint samples}

The analytical results obtained for the two reference paint layers, and for the $16^{\text {th }}$ century gilding sample and for the paint tube of Edward Munch are reported in Table 5. The chromatograms are shown in Figures 3 , and 4 and 5 . The table reports the total amount of free fatty acids (FFAs) - determined by derivatization with HMDS as the sum of the content of the quantified acids (lauric, myristic, palmitic, oleic, stearic, suberic, azelaic, and sebacic acids) normalised for the sample weight- and the total amount of metal soaps and free fatty acids (metal soaps + FFAs), determined by derivatization with BSTFA as the sum of the content of the quantified acids normalised for the sample weight.

The ratio between the content of azelaic acid and palmitic acid $(A / P)$ and the ratio between the content of palmitic acid and stearic acid $(P / S)$ are also reported, as they are common parameters used in the characterisation of a lipid material in a painting [41]. The total amount of fatty and di-acids with respect to the oil content, both hydrolysed and saponified, as well as the relative contents of palmitic and stearic acids are also reported.

Table 5 - GC/MS data relative to the paint layers containing vine black (VB) and lead white (LW) as pigments, and for the gilding sample. A/P: azelaic acid/palmitic acid; P/S: palmitic acid/stearic acid

\begin{tabular}{c|c|c|c|c|c|c}
\hline \multicolumn{2}{c|}{} & $A / P$ & $P / S$ & $\begin{array}{c}\text { Weight \% } \\
\text { (sample weight) }\end{array}$ & $\begin{array}{c}\text { Weight \% } \\
\text { (organic content) }\end{array}$ & $\begin{array}{c}\text { P and S weight \% } \\
\text { (organic content) }\end{array}$ \\
\hline $\begin{array}{c}\text { Paint sample } \\
\text { LW }\end{array}$ & FFAs & - & - & - & - & - \\
\cline { 2 - 7 } & metal soaps + FFAs & $0.8 \pm 0.2$ & $0.7 \pm 0.3$ & $3 \%$ & $23 \%$ & $17 \%$ \\
\hline $\begin{array}{c}\text { Paint sample } \\
\text { VB }\end{array}$ & FFAs & $2.7 \pm 0.6$ & $1.4 \pm 0.2$ & $2 \%$ & $5 \%$ & $2 \%$ \\
\cline { 2 - 7 } $\begin{array}{c}\text { Gliding } \\
\text { sample }\end{array}$ & metal soaps + FFAs & $2.3 \pm 0.3$ & $1.4 \pm 0.3$ & $2 \%$ & $5 \%$ & $2 \%$ \\
\hline \multirow{2}{*}{$\begin{array}{c}\text { Munch paint } \\
\text { tube }\end{array}$} & FFAs & 0.1 & 1.0 & $0.1 \%$ & - & - \\
\cline { 2 - 7 } & metal soaps + FFAs & 0.8 & 0.7 & $0.6 \%$ & - & - \\
\hline
\end{tabular}

The paint sample from the reference paint layer containing lead white (LW) showed the presence of free fatty acids below the limit of detection, and traces of dicarboxylic acids (below the quantitation limit), while metal soaps were present and above the quantitation limit. This result is in accordance with what could be expected from a paint sample containing a drying oil and lead white: during ageing, free fatty acids have reacted quantitatively with lead, to produce the corresponding metal soaps [10, 12-14]. The paint layer containing vine black (VB), which is a pigment mainly comprised of carbon [42], contained only free fatty acids, while metal soaps were absent, as it must be expected from a paint sample not containing metals.

The same paint layers were analysed in a previous work, and the relative content of oil with respect to the sample weight was determined: the vine black paint contains $34 \%$ of oil, while the lead white one contains $14 \%$ of oil [43]. The data in Table 5 show that the sample containing lead white has a total content of palmitic and stearic acids which is about $17 \%$ of the oil content of the paint. This value is much less in the vine black sample (2\%). This result is very interesting: the formation of metal soaps is commonly believed 
to be the consequence of the hydrolysis of the oil, followed by reaction with the pigment: preliminary experiments on model systems showed that lead compounds reacted with free fatty acids within half a month, while in the same amount of time no metal soaps were formed between lead white and triglycerides [10]. Our data showed that the relative amount of fatty acids saponified in the lead white paint was much higher than the amount of free fatty acids present the vine black paint. Both paints were prepared with the same oil at the same time, and have been conserved since then in the same conditions. A possible explanation is that when glycerides are hydrolysed in the lead white containing paint, these react promptly with lead to form metal soaps. Metal soaps then, and not lead white, catalyses further hydrolysis, followed by other saponification reactions. Another possible hypothesis is that lead soaps are formed by direct reaction between the triglycerides and lead white, although this reaction is much slower than the reaction between lead white and free fatty acids [10].

Another interesting aspect relates to the $\mathrm{A} / \mathrm{P}$ and $\mathrm{P} / \mathrm{S}$ ratios. The values obtained for the vine black sample relative to the hydrolysed fraction (Table 5) are not significantly different from those obtained from the bulk of the paint, after saponification, hydrolysis and derivatization: $A / P: 2.1 ; P / S: 1.3[43]$. This suggests that the hydrolysis rate of glycerides does not appear to be affected by the nature of the fatty acid. As for the lead white paint, the values obtained for the saponified fraction (Table 5) are significantly different with respect to those obtained from the bulk of the sample A/P:2.8; P/S: 1.3 [43]: This suggests that saponification rates depend on the nature of the acid. 

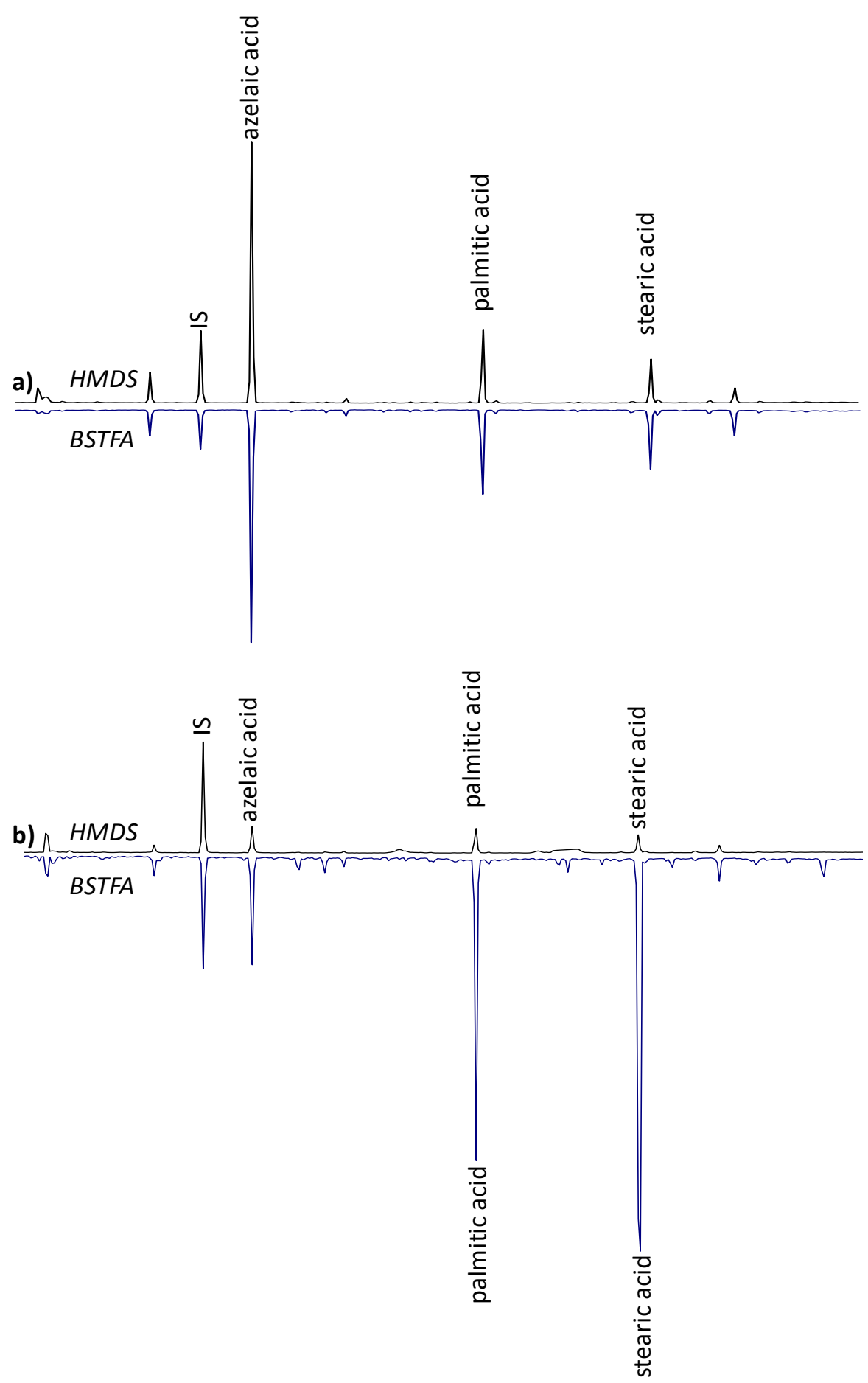

Figure $3-G C / M S$ chromatograms obtained with both the derivatization procedures for a) paint sample containing vine black (VB); b) paint sample containing lead white (LW)

The organic composition of the sample taken from a $16^{\text {th }}$ century marble sculpture by an Italian anonymous artist from Lazio was characterized by means of Py-GC/MS and FTIR in ATR mode (Detailed data on the composition are reported in the Supplementary Material). Results showed the presence of a relatively fresh drying oil and egg in the mordant for the gold leaf. The OM images suggested the presence of protrusions typical of lead white soaps and FTIR identified the presence of lead carboxylates $[10,19]$. The application of 
the new analytical approach allowed us to highlight significant differences between the hydrolyzed and the saponified fractions (Figure 4 and Table 5). Small relative amounts of diacids were present in the free form, while soaps of dicarboxylic acids and hydroxy dicarboxylic acids were relatively abundant. This suggests that in a lead containing oil paint, diacids can contribute to the formation of a ionomeric network in which they are coordinated by lead in a metal coordinated 3D network [10], together with a ionomeric network containing metal soaps of the cross linked fatty acids originating from the original polyunsaturated fatty acids [19].

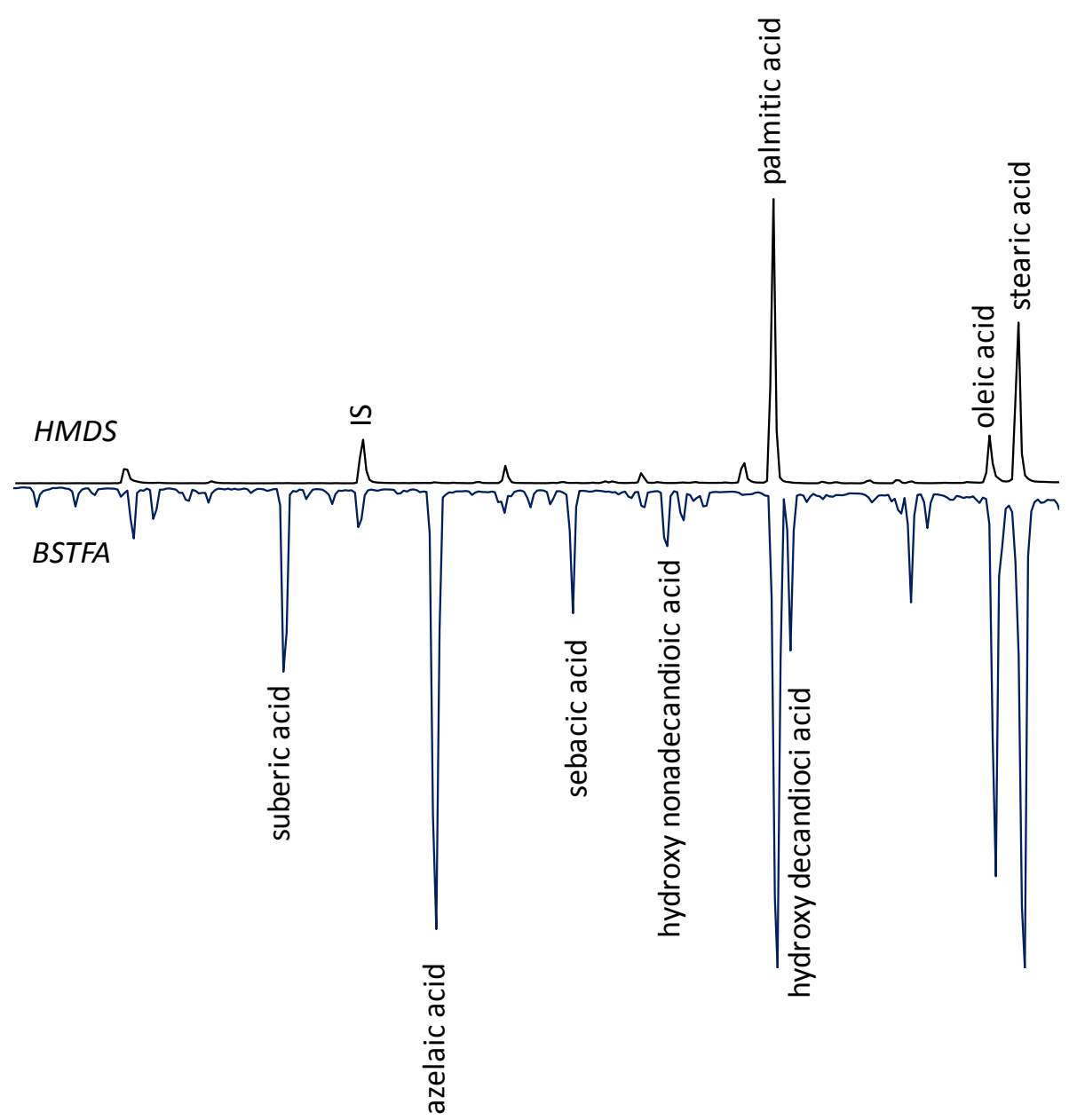

Figure 4-GC/MS chromatograms obtained with the two derivatization procedures for the gilding sample from a 16th century marble sculpture by an anonymous artist from Lazio

Finally it is important to stress that the presence of pigments, of the metal leaf, and the proteinaceous material did not hinder the derivatization reactions, as the internal standard added in the analyses (tridecanoic acid) was quantitatively derivatized $(\mathrm{CV}<15 \%)$.

The organic composition of the sample from the paint tube used by Edvard Munch, was previously characterized by HPLC-ESI-Q-Tof, and the results showed the presence of a mixture of drying oils (linseed 
and walnut oil). The FTIR spectrum did not show absorption bands ascribable to metal carboxylates. The results obtained with the new analytical approach are reported in Figure 5 and Table 5.



Figure $5-\mathrm{GC} / \mathrm{MS}$ chromatograms obtained with the two derivatization procedures for the Edvard Munch paint tube sample

The A/P ratio for both fractions was around 0.7 , while the $\mathrm{P} / \mathrm{S}$ ratio was lower for the fraction metal soaps + FFAs with respect to the fraction FFAs (3.1 versus 4.3). This enabled us to estimate the presence of about $1 \%$ of carboxylates, among which stearates were the major constituents, suggesting that the paint formulation was added with small amounts of metal stearates, which could not be detected by FTIR, as they were below the detection limit of the technique. 


\section{Conclusions}

This work presents the development of a new analytical approach, based on the use of two different silylating procedures, for the GC/MS analysis of mixtures of free fatty acids (based on the use of HMDS) and fatty acid carboxylates (based on the use of BSTFA).

The reaction conditions for the derivatization of fatty acid carboxylates were optimized using a full factorial experimental design. The new procedure was applied to a set of reference metal carboxylates with different metal ions, showing quantitative reaction yields. The proposed analytical method was validated following the ICH guidelines, and proved to be extremely sensitive and selective.

The new analytical approach demonstrated to be suitable for the characterization of mixtures of free fatty acids and metal soap in paint samples characterized by an increasing complexity. Results showed that this procedure constitutes a useful tool to help us understand the molecular composition of oil paints, supporting the study of molecular changes undergone by the paint constituents upon curing and ageing.

\section{Acknowledgment}

This work was performed within the context of the JPI CMOP project: "Cleaning of modern oil paint" (Heritage Plus Joint Call project 2015-2018). Moreover, this work has received funding from University of Pisa grant PRA_2016_13 “Analytical chemistry applications for deepening the knowledge of materials and techniques in modern and contemporary art".

The authors wish to express their thanks to Prof. Gennaro Pescitelli (Department of Chemistry and Industrial Chemistry, University of Pisa, Italy) for the fruitful discussions, Prof. Leslie Carlyle (REQUIMTE, Lisbon, Portugal), Biljana Topalova-Casadiego (Munch Museum, Oslo), Eva Storevik Tveit (Munch Museum, Oslo), and Hartmut Kutzke (Museum of Cultural History, Oslo) for kindly providing us some of the paint samples analysed in this work. 


\section{References}

[1] M.C. Corbeil, L. Robinet, X-ray powder diffraction data for selected metal soaps, Powder Diffraction, 17 (2002) 52-60. [2] J. Li, Y. Wu, Lubricants in Pharmaceutical Solid Dosage Forms, Lubricants, 2 (2014) 21.

[3] A. Burnstock, K.J.v.d. Berg, S.D. Groot, L. Wijnberg, Reprints of the Modern Paints Uncovered conference, in: T. Learner (Ed.), Los Angeles, 2008, pp. 177-188.

[4] M. Gönen, T.O. Egbuchunam, D. Balköse, F. Inal, S. Ülkü, Preparation, Characterization and Applications of Magnesium Stearate, Cobalt Stearate and Copper Stearate, in: D. Balköse, D. Horak, L. Šoltés, A.K. Haghi, G.E. Zaikov (Eds.) Key Engineering Materials, Apple Academic Press2014, pp. 209-214.

[5] J. La Nasa, M. Zanaboni, D. Uldanck, I. Degano, F. Modugno, H. Kutzke, E.S. Tveit, B. Topalova-Casadiego, M.P. Colombini, Novel application of liquid chromatography/mass spectrometry for the characterization of drying oils in art: Elucidation on the composition of original paint materials used by Edvard Munch (1863-1944), Analytica Chimica Acta, 896 (2015) 177-189.

[6] C.S. Tumosa, A Brief History of Aluminum Stearate as a Component of Paint, Waac newsletter, 23 (2001).

[7] G. Osmond, Zinc white: a review of zinc oxide pigment properties and implications for stability in oil-based paintings, AICCM bulletin, 33 (2012) 20-29.

[8] G. Osmond, K. Keune, J. Boon, A study of zinc soap aggregates in a late 19th century painting by RG Rivers at the Queensland Art Gallery, AICCM bulletin, 29 (2005) 37-46.

[9] K. Keune, A. van Loon, J.J. Boon, SEM backscattered-electron images of paint cross sections as information source for the presence of the lead white pigment and lead-related degradation and migration phenomena in oil paintings, Microscopy and Microanalysis, 17 (2011) 696-701.

[10] K. Keune, J.J. Boon, Analytical imaging studies of cross-sections of paintings affected by lead soap aggregate formation, Studies in Conservation, 52 (2007) 161-176.

[11] G. Silvester, A. Burnstock, L. Megens, T. Learner, G. Chiari, K.J. van den Berg, A cause of water-sensitivity in modern oil paint films: the formation of magnesium sulphate, Studies in Conservation, 59 (2014) 38-51.

[12] J.J. Boon, J. van der Weerd, K. Keune, P. Noble, J. Wadum, Mechanical and chemical changes in Old Master paintings: dissolution, metal soap formation and remineralization processes in lead pigmented ground/intermediate paint layers of 17th century paintings, 13th Triennial Meeting of the ICOM Committee for Conservation in Rio De Janeiro Preprints (R. Vontobel ed), James \& James, London. p. 401, 2002.

[13] M. Cotte, E. Checroun, J. Susini, P. Dumas, P. Tchoreloff, M. Besnard, P. Walter, Kinetics of oil saponification by lead salts in ancient preparations of pharmaceutical lead plasters and painting lead mediums, Talanta, 70 (2006) 11361142.

[14] M. Cotte, E. Checroun, W. De Nolf, Y. Taniguchi, L. De Viguerie, M. Burghammer, P. Walter, C. Rivard, M. Salomé, K. Janssens, J. Susini, Lead soaps in paintings: Friends or foes?, Studies in Conservation, 62 (2017) 2-23.

[15] L. Robinet, M.-C. Corbeil-a, The Characterization of Metal Soaps, Studies in Conservation, 48 (2003) 23-40.

[16] V. Otero, D. Sanches, C. Montagner, M. Vilarigues, L. Carlyle, J.A. Lopes, M.J. Melo, Characterisation of metal carboxylates by Raman and infrared spectroscopy in works of art, Journal of Raman Spectroscopy, 45 (2014) 11971206.

[17] R. Mazzeo, S. Prati, M. Quaranta, E. Joseph, E. Kendix, M. Galeotti, Attenuated total reflection micro FTIR characterisation of pigment-binder interaction in reconstructed paint films, Analytical and Bioanalytical Chemistry, 392 (2008) 65-76.

[18] L. Robinet, M.C. Corbeil, The Characterization of Metal Soaps, Studies in Conservation, 48 (2003) 23-40.

[19] J.J. Hermans, K. Keune, A. van Loon, R.W. Corkery, P.D. ledema, lonomer-like structure in mature oil paint binding media, RSC Advances, 6 (2016) 93363-93369.

[20] J.J. Hermans, K. Keune, A.v. Loon, P.D. ledema, The crystallization of metal soaps and fatty acids in oil paint model systems, Physical Chemistry Chemical Physics, 18 (2016) 10896-10905.

[21] J.J. Hermans, K. Keune, A. van Loon, P.D. ledema, An infrared spectroscopic study of the nature of zinc carboxylates in oil paintings, Journal of Analytical Atomic Spectrometry, 30 (2015) 1600-1608.

[22] M. Cotte, E. Checroun, J. Susini, P. Walter, Micro-analytical study of interactions between oil and lead compounds in paintings, Applied Physics A, 89 (2007) 841-848.

[23] M.G. MacDonald, M.R. Palmer, M.R. Suchomel, B.H. Berrie, Reaction of Pb (II) and Zn (II) with Ethyl Linoleate To Form Structured Hybrid Inorganic-Organic Complexes: A Model for Degradation in Historic Paint Films, ACS Omega, 1 (2016) 344-350.

[24] S. Zumbühl, N.C. Scherrer, W. Müller, Derivatisation technique for infrared spectroscopy-characterisation of oxidative ageing products in modern oil paint, Issues in Contemporary Oil Paint, Springer2014, pp. 213-225.

[25] F.C. Izzo, K.J.v.d. Berg, H.v. Keulen, B. Ferriani, E. Zendri, Modern Oil Paints - Formulations, Organic Additives and Degradation: Some Case Studies, in: J.K. van den Berg, A. Burnstock, M. de Keijzer, J. Krueger, T. Learner, d.A. Tagle, G. Heydenreich (Eds.) Issues in Contemporary Oil Paint, Springer International Publishing, Cham, 2014, pp. 75-104.

[26] J.D.J.v.d. Berg, K.J.v.d. Berg, J.J. Boon, Determination of the degree of hydrolysis of oil paint samples using a twostep derivatisation method and on-column GC/MS, Progress in Organic Coatings, 41 (2001) 143-155.

[27] P. Dietemann, U. Baumer, C. Bell, I. Fiedler, The Binding Media of Cologne Painting in the First Half of the Fifteenth Century, Zeitschrift für Kunsttechnologie und Konservierung, 26 (2012) 286-290.

[28] J. Koller, F. Irene, U. Baumer, Die Bindemittel auf Dürers Tafelgemälden, Albrecht Dürer-Die Gemälde der Alten Pinakothek. Bayerische Staatsgemäldesammlungen, München, (1998) 102-119.

[29] L. Carlyle, Molart Fellowship Report: Historical Reconstructions of Artists's Oil Paint: an investigation of oil processing methods and the use of medium- modifiers. Report n 72894, Canadian Conservation Institute2000. 
[30] V. Otero, D. Sanches, C. Montagner, M. Vilarigues, L. Carlyle, J.A. Lopes, J.M. Melo, Characterisation of metal carboxylates by Raman and infrared spectroscopy in works of art, Journal of Raman Spectroscopy, 45 (2014) 11971206.

[31] A. Andreotti, I. Bonaduce, M.P. Colombini, G. Gautier, F. Modugno, E. Ribechini, Combined GC/MS Analytical Procedure for the Characterization of Glycerolipid, Waxy, Resinous, and Proteinaceous Materials in a Unique Paint Microsample, Analytical Chemistry, 78 (2006) 4490-4500.

[32] Public paintings by Edvard Munch and his contemporaries: change and conservation challenges, Archetype Publications, London, 2015.

[33] I. Bonaduce, E. Ribechini, F. Modugno, M.P. Colombini, Analytical approaches based on gas chromatography mass spectrometry (GC/MS) to study organic materials in artworks and archaeological objects, Topics in Current Chemistry, 2016, pp. 1-37.

[34] S.C. Moldoveanu, V. David, Modern Sample Preparation for Chromatography, Elsevier Science2014.

[35] R. Evershed, Advances in silylation, Handbook of derivatives for chromatography, 2 (1993) 51-108.

[36] H.D. Burrows, V.M. Lobo, 3 Potentiometric Study on the Interactions between Divalent Cations and Sodium Carboxylates in Aqueous Solution, in: Gennady E. Zaikov, Alexander N. Goloshchapov, A.V. Lobano (Eds.) Progress in Organic and Physical Chemistry: Structures and Mechanisms, Apple Academic Press, Toronto, New Jersey, 2013, pp. 23.

[37] R.G. Brereton, Chemometrics: Data Analysis for the Laboratory and Chemical Plant, Wiley2003.

[38] Wolfram|Alpha, Wolfram Alpha LLC, 2017.

[39] M.J. Plater, B. De Silva, T. Gelbrich, M.B. Hursthouse, C.L. Higgitt, D.R. Saunders, The characterisation of lead fatty acid soaps in 'protrusions' in aged traditional oil paint, Polyhedron, 22 (2003) 3171-3179.

[40] I. Degano, J. La Nasa, E. Ghelardi, F. Modugno, M.P. Colombini, Model study of modern oil-based paint media by triacylglycerol profiling in positive and negative ionization modes, Talanta, 161 (2016) 62-70.

[41] I. Bonaduce, E. Ribechini, F. Modugno, M.P. Colombini, Analytical approaches based on gas chromatography mass spectrometry (GC/MS) to study organic materials in artworks and archaeological objects, Topics in Current Chemistry, 374 (2016) 6.

[42] G. Buxbaum, Industrial Inorganic Pigments, Wiley2008.

[43] I. Bonaduce, L. Carlyle, M.P. Colombini, C. Duce, C. Ferrari, E. Ribechini, P. Selleri, M.R. Tine, New insights into the ageing of linseed oil paint binder: a qualitative and quantitative analytical study, PLoS One, 7 (2012) e49333. 